\title{
Steroid Psychosis: A Case Series of Three Patients
}

\author{
Vineetha Bharathan Menon ${ }^{1 *}$, Ansu Anie Sunny ${ }^{1}$, Pratibha Pereira², Shashidhara Chikkaveeraiah², \\ Madhan Ramesh ${ }^{1}$
}

1Department of Pharmacy Practice, JSS College of Pharmacy, JSS University, Mysore- 15, Karnataka, INDIA.

${ }^{2}$ Department of Internal Medicine, JSS Hospital and Medical College, JSS University, Mysore- 15, Karnataka, INDIA.

\begin{abstract}
Background: Steroids are widely used in modern medicine for the treatment of various ailments. Steroid psychosis is a known complication of steroid therapy, although infrequent. Herein we describe a series of three cases where patients presented with pure psychosis without mood disturbance, which is highly unlikely, following steroid therapy. Cases: Two female and one male patients aged 23 years, 50 years and 80 years respectively developed psychosis following steroid administration. In the first case, oral methylprednisolone (48 $\mathrm{mg} /$ day) was used in the management of systemic lupus erythematosus, second case received intravenous dexamethasone (36 mg/day) for the management of bronchopneumonia, while third case had a two day course of intravenous methylprednisolone $(120 \mathrm{mg} / \mathrm{day})$ for the treatment of chronic obstructive pulmonary disease. Conclusion: Consistent with the previous studies, female gender and high steroid dose were identified as the common risk factors for the development of steroid psychosis. Steroid psychosis is a major complication, which if recognized early can be effectively treated. Treatment with high dose of steroids should be used cautiously to avoid psychotic adverse reactions.
\end{abstract}

Key words: Adverse drug reaction, Case series, Corticosteroids, Psychosis.

\section{INTRODUCTION}

Corticosteroids have been used effectively for years to treat a wide variety of both acute and chronic medical conditions, ${ }^{1}$ ranging from autoimmune and system disorders to severe inflammatory and allergic reactions, as well as in palliative care and support. ${ }^{2}$ Although a powerful therapeutic option, corticosteroids are associated with serious adverse effects, both physiologic like hypertension, Cushing's syndrome, hypernatremia, hypokalemia, peptic ulcer disease and psychiatric like depression, euphoria. While the somatic adverse effects of corticosteroid therapy have been extensively researched and widely described, the neuropsychiatric adverse effects have received less attention. ${ }^{3}$ Herein, we report three cases of psychosis secondary to steroid use in patients with no previous history of psychosis.

\section{CASE SERIES}

\section{Case 1}

A 23-year-old female was admitted to the internal medicine ward of a tertiary care hospital with high grade fever associated with chills and rigors since 10 days and generalized weakness since seven days. Her past medical history was found to be insignificant. On clinical examination she was afebrile and had palpable purpura on both elbows, palms and tip of fingers and butterfly rash on face. Her blood pressure and pulse rate at admission was found to be $110 / 70 \mathrm{mmHg}$ and 84 beats/min. On examination, all the body systems were found to be clinically stable. Hemoglobin was $11.4 \mathrm{gm} / \mathrm{dl}(12-15 \mathrm{gm} / \mathrm{dl})$, TLC was 3000 cells/cumm (4000-11,000 cells/cumm), PCV 33.8\% (36-46\%), PLT $0.87 \mathrm{lakh} / \mathrm{cumm}$ (1.5-4 lakh/cumm), MCV $77.7 \mathrm{fl}$ (83-101 fl), MCH $26.2 \mathrm{pg}$ (27-32 pg) and ESR $60 \mathrm{~mm} / \mathrm{hr}(20 \mathrm{~mm} /$ hr). PBS was suggestive of leucopenia with
DOI: 10.5530/ijopp.11.1.9

Address for correspondence: Dr. Vineetha Bharathan Menon,

Doctoral Research Scholar, Department of Clinical Pharmacy, JSS Medical College and Hospital and Department of Pharmacy Practice, JSS College of Pharmacy, JSS University, Mysore- 15, Karnataka, INDIA. Phone no: 091-953-828-3470 Email Id: vineethamenon54@ yahoo.com

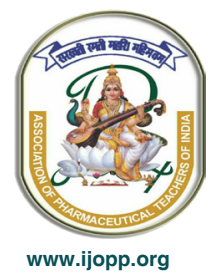


thrombocytopenia. ANA was positive and antibodies to Nrnp, Sm and Rib.P-protein were detected. Based on the subjective and objective evidence she was diagnosed with emerging lupus.

During the four days of hospitalization she was put on oral pantoprazole $(40 \mathrm{mg} /$ day), oral methylprednisolone (48 mg/day), oral doxycycline (200 mg/day), oral folic acid $(5 \mathrm{mg} /$ day) and multivitamins. On discharge the above medications were continued for another 15 days in addition to oral hydroxychloroquine $(200 \mathrm{mg} /$ day for 15 days). Within a week of treatment, patient was brought back to the hospital with complaints of irritability, irrelevant talking, decreased sleep and appetite. She had no past personal or family history of psychiatric disorders. She was diagnosed with steroid associated psychosis and methylprednisolone was implicated as the causative drug. Methylprednisolone was tapered (24 mg/day) and she was put on risperidone and olanzapine. After a month, during follow up, there was improvement in her symptoms. Over the next three months the dose of methylprednisolone was tapered and eventually stopped. On follow up at the end of three months, patient's psychiatric manifestations had completely disappeared.

The Naranjo's criteria and World Health Organization (WHO) probability scale were applied to determine the causality for suspected adverse drug reaction (ADR). The causality assessment with both scales revealed that the ADR due to methylprednisolone in this case was 'probable' (Naranjo overall score: 6). Severity of the ADR was evaluated using the Modified Hartwig and Siegel scale, based on which it was categorized as moderate (level 4(b)) reaction.

\section{Case 2}

A 50-year-old female was admitted to the internal medicine ward of a tertiary care hospital with fever and chills, headache and generalized weakness since five days. Her past medical history was insignificant. She also had no history of known drug allergies. Her body temperature was $98^{\circ} \mathrm{F}$, heart rate 76 beats $/ \mathrm{min}$, respiratory rate 17 cycles/min and blood pressure 130/80 mmHg. Following admission, she was diagnosed with viral fever, bronchopneumonia, acute kidney injury and multiple organ dysfunction syndrome and was put on intravenous piperacillin/tazobactum (6.75 g per day), intravenous clindamycin (900 mg per day), oral acetaminophen (1950 $\mathrm{mg}$ per day), intravenous ondansetron (4 $\mathrm{mg}$ as required), intravenous esomeprazole (40 $\mathrm{mg}$ per day), salbutamol/ ipratropium bromide inhalation $(3.75 \mathrm{mg} / 1500 \mathrm{mcg}$ per day), normal saline infusion (at a rate of $75 \mathrm{cc} / \mathrm{h}$ ), intravenous artesunate ( $240 \mathrm{mg}$ per day), and intravenous dexamethasone (8 $\mathrm{mg}$ stat followed by $36 \mathrm{mg}$ per day). Patient was symptomatically better by the next day, however on the fourth day of admission, she was found to be anxious and agitated and had behavioral changes. She had bizarre behavior, auditory and visual hallucinations. Her mental status examination revealed irritability, agitation, auditory and visual hallucinations, delusions, disorganized speech and thinking. Her relatives informed negative past psychiatric history, including depressive, maniac or psychotic symptoms. There was also no family history of psychiatric disorders. She was diagnosed to have steroid induced psychosis. Dexamethasone was suspected as the causative drug and was tapered and withdrawn over the next two days. After four days, she was relieved from her symptoms and her thought form and content were normal. Her psychotic findings like agitation, delusions and hallucinations also completely disappeared. She was discharged after seven days.

The Naranjo's criteria and WHO probability scale were applied to determine the causality for suspected ADR. The causality assessment with both scales revealed that the ADR due to dexamethasone in this case was 'probable' (Naranjo overall score: 6). Severity of the ADR was evaluated using the Modified Hartwig and Siegel scale, based on which it was categorized as moderate (Level 4(a)) reaction.

\section{Case 3}

An 80-year-old male patient, with body mass index of $35 \mathrm{~kg} / \mathrm{m}^{2}$, was admitted to the internal medicine unit of a tertiary care hospital with complains of breathlessness and cough with expectoration since three days. He also had complains of orthopnea. He was a known case of chronic obstructive pulmonary disease since 10 years and was on oral theophylline (500 $\mathrm{mg}$ per day) and inhaler levosalbutamol/ipratropium bromide combination (1.25 $\mathrm{mg} / 500 \mathrm{mcg}$ as required). He was also a known case of umbilical hernia, acid peptic disease and gastritis, medication history of which were not known. His social history was suggestive that he was a smoker for the past 40-45 years but had quit 5 years back. He was a nonalcoholic.

On examination, his pulse respiratory rate and blood pressure were 95 beats/min, 44 cycles/min and $140 / 90 \mathrm{mmHg}$ respectively. Wheezing was present with bilateral extensive rhonchi in all respiratory areas. Other body systems were found to be within normal limits. All hematological and biochemical tests came out unremarkable. Based upon the subjective and objective findings his diagnosis was made as acute exacerbation 
of chronic obstructive pulmonary disease, accelerated hypertension and query of cardiac asthma. Following admission, he was put on intravenous ceftriaxone ( $2 \mathrm{gm}$ per day), levosalbutamol/ipratropium bromide inhalation (1.25mg/500 mcg Q4h), budesonide inhalation (0.5 $\mathrm{mg}$ Q6h), intravenous methylprednisolone (125 mg STAT followed by $120 \mathrm{mg}$ per day) and intravenous nitroglycerine ( $5 \mathrm{mcg}$ per min which was tapered accordingly), intravenous furosemide (40 $\mathrm{mg}$ STAT), oral azithromycin (500 $\mathrm{mg}$ per day), and oral doxofylline (800 $\mathrm{mg}$ per day). On the third day of treatment, patient developed agitated and aggressive personality. He had psychotic symptoms with auditory and visual hallucination. He started speaking to himself and stated that he is a God and had come to save the humanity. He stated seeing images not seen by others. His mood was found to be elevated. The mental status examination showed that the patient had delirium. On enquiry, his care takers denied the patient having any previous history of psychiatric disorders. There was also no family history of psychiatric illness. Patient was diagnosed with steroid induced psychosis and methylprednisolone dose was tapered and stopped over the next two days. Two days after stopping the drug, patients' symptoms improved dramatically. No psychotic symptoms were further observed at any time during his hospital stay and patient was discharged after eight days of hospitalization.

Causality was assessed by using both Naranjo's criteria and the WHO probability scale, which revealed a 'possible' (score 4) causal relationship between the drug and the adverse event. Severity of the reaction was evaluated using the Modified Hartwig and Siegel scale and was categorized as moderate (level 4 (a) reaction) in its severity.

\section{Informed Consent}

Voluntary approval were obtained from all the three patients and the case series was prepared in accordance with the Declaration of Helsinki.

\section{DISCUSSION}

According to literature, the incidence of steroid induced psychosis varies widely between $13-62 \%$, with a weighted average of $27.6 \%$. Vast majority of these reactions are mild to moderate. The incidence of a severe psychiatric syndrome ranges between $1.6 \%$ and $50 \%$, with a weighted average of $5.7 \%{ }^{4}$ The etiology and pathogenesis of psychiatric effects of steroids are poorly understood. High levels of glucocorticoids are known to increase the dopamine levels which may have several psychiatric implications. Conversely, steroids have also been linked with decreased peripheral and central serotonin secretions. It has been demonstrated that serotonergic afferent neurons are direct inhibitors of dopamine release at dopaminergic axons. Both these correlations may have implications with regard to possible prophylaxis of steroid psychosis. ${ }^{1}$ Corticosteroids are also associated with changes in the temporal lobe, detected by structural, functional, and spectroscopic imaging. ${ }^{2}$ Neuropsychiatric adverse effects of corticosteroids are complex, unpredictable and often severe, ranging across most categories of psychopathology. Clinical signs commonly associated with steroid induced psychosis comprises of visual and auditory hallucinations, delusional thinking, paranoia, affective disturbances (depression, apathy, hypomania, panic), depersonalization, motor disturbances (over activity, immobility), aggressive behavior and cognitive impairment. However, many publications on this topic appear to support the symptoms of depressive disorder $(40 \%)$ in majority of patients followed by mania $(25 \%) ., 5$ Interestingly, all our patients presented with symptoms of psychosis without any mood disturbance.

Gender seems to be a risk factor to develop steroid psychosis. Females have minimal but statistically significant, increased risk for psychiatric disturbances than males. ${ }^{24,5}$ Similarly, we found higher incidence of steroid psychosis in females compared to males as 2 of our patients were females. Dosage was also found to be directly related to the incidence of adverse effects, high dose being a primary risk factor for psychosis. The Boston Collaborative Drug Surveillance Program reported that among patients taking prednisone, psychiatric disturbances are seen in $1.3 \%$ of patients taking $<40 \mathrm{mg} /$ day, $4.6 \%$ of patients taking $40-80 \mathrm{mg} /$ day and $18.4 \%$ of patients taking $>80 \mathrm{mg} /$ day. ${ }^{2,4}$ While $36 \mathrm{mg}$ of dexamethasone dose is equivalent to $240 \mathrm{mg}$ of prednisone, $120 \mathrm{mg}$ and $48 \mathrm{mg}$ of methylprednisolone is equivalent to 150 $\mathrm{mg}$ and $60 \mathrm{mg}$ of prednisone respectively. Studies has also found that the average daily dose of steroids for patients who developed psychosis was $59.5 \mathrm{mg} /$ day of prednisone or equivalent as compared with $31.1 \mathrm{mg} /$ day for patients who did not develop adverse psychiatric effects. ${ }^{6}$ Hence, it is clear that high steroid dose could also have contributed to the precipitation of psychotic symptoms in these patients.

Corticosteroid-induced psychotic symptoms frequently occur early during steroid therapy, usually within the first few weeks of therapy (median time to onset being 3-4 days). ${ }^{6,7}$ In our patients, the psychotic symptoms developed within a week of administration of the drug. The evidence suggesting causation is that the patients' mental status were functioning well before initiating the steroids and none of the other drugs they received 
can precipitate psychosis. Studies state that premorbid personality, a history of previous psychiatric disorder or a history of a previous steroid psychosis can increase a patient's risk of developing a psychotic reaction. ${ }^{4}$ However, none of our patients had any past medical history of psychiatric disorders. Other risk factors to develop steroid psychosis include hypoalbuminuria and use of drugs which can increase circulating levels of corticosteroid like cytochrome P450 enzyme inhibitor. ${ }^{2,5}$ In the first two cases use of cytochrome P450 enzyme inhibitors like doxycycline, hydroxychloroquine, pantoprazole and esomeprazole could have also contributed to the development of psychosis. ${ }^{8,9,10,11}$

Various treatment approaches are available for steroid induced psychosis. The most widely used and effective treatment strategy is to taper and discontinue steroids where possible. In some cases this has proven sufficient in reversal of symptoms. Another option is to treat the patient with antipsychotic medications. ${ }^{2,4} \mathrm{~A}$ review of literature conducted by Lewis and Smith suggested that tapering of steroid alone was found to be effective in more than $90 \%$ of cases. However, they found 100\% effectiveness in cases where neuroleptics and steroid taper, or treatment with lithium alone, or treatment with ECT alone was initiated. ${ }^{5,12}$ In two of our patients, the psychotic symptoms resolved soon after drug tapering and withdrawal, without initiation of any psychotropic medications. However, one patient received treatment with antipsychotics like risperidone and olanzapine. Corticosteroids dexamethasone and methylprednisolone have half-life of 36-54 $\mathrm{h}$ and 18-36 $\mathrm{h}$ respectively. ${ }^{5}$ This may have led to persistence of symptoms even after drug withdrawal.

\section{CONCLUSION}

This case report adds to the growing evidence supporting the existence of steroid induced psychotic symptoms that range across a spectrum of symptoms from anxiety and insomnia to severe mood disorders and psychosis. As the reaction is probably iatrogenic and often preventable with cautious use of steroids, clinical practice could benefit from awareness of these adverse effects, risk factors and possible treatment options. Physicians should educate the patients and their family about the risk of steroid induced psychiatric adverse effects prior to initiating the treatment.

\section{CONFLICT OF INTEREST}

The authors report no conflict of interest that is directly relevant to the content of the case series.

\section{ACKNOWLEDGEMENT}

Authors would like to thank JSS University for the constant support and encouragement.

\section{ABBREVIATIONS USED}

ADR: Adverse Drug Reaction; WHO: World Health Organisation

\section{SUMMARY}

Prescribed steroids are reported to cause psychiatric symptoms including psychosis, often within weeks of treatment. Thus healthcare providers should be aware of the potential adverse reactions of steroid therapy. Knowledge of risk factors and effective treatment reduce severity of these reactions. Tapering or discontinuation of the drug help to relieve the psychiatric adverse reactions, although psychotropic medications are needed in most cases.

\section{REFERENCES}

1. French J, Khan A, White H. Steroid induced psychosis in an asthmatic child: case report and 10-year literature review. Can Child Adolesc Psychiatr Rev. 2003;12(4):117-8.

2. Airagnes G, Rouge-Maillart C, Garre JB, Gohier B. Homicide and associated steroid acute psychosis: a case report. Case Reports in Medicine. 2011;4p.

3. Warrington TP, Bostwick JM. Psychiatric adverse effects of corticosteroids. Mayo Clin Proc. 2006;81(10):1361-7.

4. Hall RCW. Psychiatric adverse drug reactions: steroid psychosis.

5. Kenna HA, Poon AW, De Los Angeles P. Psychiatric complications of treatment with corticosteroids: review with case report. Psychiatry and Clinical Neurosciences. 2011;65(6): 549-60.

6. Milanlioglu A, Gulec M. Risperidone treatment in a steroid induced psychosis case. The Journal of Psychiatry and Neurological Sciences. 2011;24(1):80-4.

7. Lyons PR, Newman PK, Saunders M. Methylprednisolone therapy in multiple sclerosis: a profile of adverse effects. Journal of Neurology, Neurosurgery and Psychiatry. 1988;51(2):285-7.

8. Cytochrome P 450. http://www.straighthealthcare.com/cytochrome-p450-2d6. $\mathrm{html}$.

9. Li XQ, Andersson TB, Ahlstrom M, Weidolf L. Comparison of inhibitory effects of the proton pump-inhibiting drugs omeprazole, esomeprazole, lansoprazole, pantoprazole, and rabeprazole on human cytochrome P450 activities. Drug Metab Dispos. 2004;32(8):821-7.

10. Medications metabolized by cytochrome P450 3A4. https://www.cpsbc.ca/files/ pdf/Methadone-Medications-Cytochrome-P450-3A4.pdf.

11. Cytochrome P450 2D6 Known Drug Interaction Chart. http://www. mayomedicallaboratories.com/it -mmfiles/Cytochrome_P450_2D6_Known_ Drug_Interaction_Chart.pdf.

12. Flores $\mathrm{BH}$, Gumina HK. The neuropsychiatric sequelae of steroid treatment. http://www.dianafoundation.com/articles/df_04_article_01_steroids_pg01.html. 